Research Paper

\title{
Impact of Tissue Enolase 1 Protein Overexpression in Esophageal Cancer Progression
}

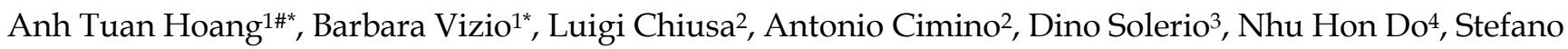 \\ Pileci $^{3}$, Michele Camandona ${ }^{3}$, Graziella Bellone ${ }^{1 凶}$ \\ 1. Department of Medical Sciences, University of Turin, 10126 Turin, Italy \\ 2. Pathology Unit, AOU City of Health and Science of Turin, 10126 Turin, Italy. \\ 3. Department of Surgical Sciences, University of Turin, Unit of Digestive and Oncological Surgery 1U, AOU City of Health and Science of Turin, 10126 Turin, \\ Italy. \\ 4. Vietnam National Institute of Ophthalmology, Hanoi, Vietnam. \\ \# Current address: Vietnam National Institute of Ophthalmology, Hanoi, Vietnam. \\ * These Authors contributed equally. \\ $\triangle$ Corresponding author: Graziella Bellone, Department of Medical Sciences, University of Turin, Via Genova 3, 10126 Turin, Italy. Tel. 39-011-6705370, Fax \\ 39-011-6705373, email: graziella.bellone@unito.it
}

(c) The author(s). This is an open access article distributed under the terms of the Creative Commons Attribution License (https://creativecommons.org/licenses/by/4.0/). See http://ivyspring.com/terms for full terms and conditions.

Received: 2020.09.01; Accepted: 2020.12.18; Published: 2021.01.26

\begin{abstract}
Enolase (ENO) 1 is a key glycolytic enzyme and important player in tumorigenesis. ENOI overexpression has been correlated with tumor progression and/or worse prognosis in several solid malignancies. However, data concerning the impact of ENOI in cancer conflict. The study correlated local and circulating ENO1 protein levels in esophageal cancer (EC) with clinicopathological data, to assess its potential clinical value. ENOI expression was analyzed by immunohistochemistry in paired tumor and non-tumor tissue samples from $40 \mathrm{EC}$ cases and mucosal biopsies from 45 Barrett's esophagus (BE) cases, plus in plasma from these patients and 25 matched healthy controls. ENOI was abnormally elevated in cancer-cell cytoplasm in both EC types, in esophageal squamous cell carcinoma and in adenocarcinoma (EAC), increasing significantly with tumor stage progression and the transition from BE to EAC. EAC patients exhibited significantly lower ENOI plasma concentrations than normal subjects. Neither local nor systemic ENOI expression levels were significantly associated with overall survival. These results indicate ENOI as potential biomarker, delineating a population of patients with Barrett's esophagus at high risk of cancer, and as new therapeutic opportunity in EC patient management. However, further confirmation might be necessary.
\end{abstract}

Key words: Enolase 1, esophageal cancer, Barrett's esophagus, tumor progression.

\section{Introduction}

Esophageal cancer (EC) ranks eighth among common cancers and sixth among all cancer-related mortality worldwide [1]. Overall survival is below $10 \%$ and, despite recent advances in therapeutic strategies, the 5-year survival rate of patients who have undergone resection is $20 \%-40 \%$ [2]. Neither systemic neoadjuvant (chemotherapy before "curative" surgery) nor adjuvant therapy (chemotherapy after "curative" surgery) have shown a substantial effect on survival [3]. Local control of EC can rarely be achieved, due to early micrometastatic spread, despite systemically-acting drugs [4].
The most common type of EC is esophageal squamous cell carcinoma (ESCC), with increasing morbidity in Western countries [5]. The other type is esophageal adenocarcinoma (EAC), which arises in the setting of Barrett's esophagus (BE), characterized by the replacement of healthy esophageal epithelium with metaplastic columnar cells [6]. The two subtypes are distinct entities, with some overlap concerning epidemiologic distribution, risk factors, pathogenesis, early tumor cell dissemination and metastasis, resulting in equally poor survival [7]. 
Table 1. Clinical, demographic and pathological features of the study population

\begin{tabular}{|c|c|}
\hline Characteristic & Number \\
\hline \multicolumn{2}{|l|}{ Age } \\
\hline Years, median (range) & $68(44-83)$ \\
\hline \multicolumn{2}{|l|}{ Gender } \\
\hline Female/Male & $10 / 30$ \\
\hline \multicolumn{2}{|l|}{ Histopathology } \\
\hline Adenocarcinoma & 25 \\
\hline Barrett's-associated adenocarcinoma & 2 \\
\hline Squamous cell carcinoma & 13 \\
\hline \multicolumn{2}{|l|}{ Histologic grade (G) } \\
\hline Well differentiated (G1) & 0 \\
\hline Moderately differentiated (G2) & 14 \\
\hline Moderately/poorly differentiated (G2/G3) & 10 \\
\hline Poorly differentiated (G3) & 16 \\
\hline Undifferentiated (G4) & 0 \\
\hline \multicolumn{2}{|l|}{ Tumor location } \\
\hline Hypopharynx- esophagus junction & 2 \\
\hline Cervical esophagus & 2 \\
\hline Upper thoracic esophagus & 1 \\
\hline Medium/Lower esophagus & 12 \\
\hline Cardias & 23 \\
\hline \multicolumn{2}{|l|}{ Tumor } \\
\hline In situ & 0 \\
\hline $\mathrm{T} 1$ & 1 \\
\hline $\mathrm{T} 2$ & 15 \\
\hline $\mathrm{T} 3$ & 19 \\
\hline $\mathrm{T} 4$ & 5 \\
\hline \multicolumn{2}{|l|}{ Lymph node metastasis } \\
\hline Absent (N-) & 10 \\
\hline Present $(\mathrm{N}+)$ & 30 \\
\hline \multicolumn{2}{|l|}{ Metastasis } \\
\hline Absent & 38 \\
\hline Present & 2 \\
\hline \multicolumn{2}{|l|}{ Staging } \\
\hline I & 1 \\
\hline II & 16 \\
\hline III & 17 \\
\hline IV & 6 \\
\hline
\end{tabular}

Enolase (ENO), also known as phosphopyruvate hydratase, was originally characterized as an enzyme involved in glycolytic metabolism [8]. Recent findings have shown that ENO1 plays an important role in several biological and pathophysiological processes [9]. In particular, upregulation of ENO1 has been reported in many different types of cancer [10-18], and is considered to be a key protein in tumorigenesis, cancer cell invasion, and metastasis $[19,20]$.

The expression levels of ENO1 in patients with EC have not yet been investigated. The study aimed to investigate the differential ENO1 protein expression in tumor and in adjacent non-cancerous tissue specimens, as well as in plasma from a series of primary EC, in BE patients and in matched healthy controls, in order to explore its clinicopathological relevance. The findings show that ENO1 is not a predictor of overall survival, but that it is a predictor of tumor progression, suggesting that it may help to select patients with more aggressive disease who may benefit from targeted therapies.

\section{Materials and Methods}

\section{EC patients}

Forty patients with EC, who had undergone esophagectomy at the Department of Surgical Sciences, University of Turin, Digestive and Oncological Surgery Unit 1U, AOU City of Health and Science of Turin, between January 2011 and June 2017, were qualified and included for our retrospective study approved by Institutional Ethics Committee of AOU City of Health and Science of Turin. None underwent anticancer treatment before entering the study, which was conducted under strict observance of the principles of the Declaration of Helsinki. Data including age, sex, tumor location and size, treatment protocol, curability, histology, tumor node metastasis (TNM), stage, and outcome were obtained from clinical and pathologic records. Surgical resections, clinical staging, and histopathologic classification were defined following the UICC-TNM system [21]. Final pathologic staging was determined for all patients. Table 1 gives clinicopathologic characteristics of the patients. Duration of follow-up was from time of surgery to death, dropout, or end of December 2019. During the follow-up period, 9 (22.5\%) tumors relapsed; 30 patients (75\%) died; 28 of these $(93.3 \%)$ died of the disease and $2(6.7 \%)$ of other causes.

\section{BE patients}

Forty-five patients with $\mathrm{BE}$, who underwent upper endoscopy and biopsies as part of routine dysplasia surveillance or evaluation of upper gastrointestinal symptoms at the Endoscopy and Intestinal Motility Service, University of Turin, Italy, were qualified and included for this study, conducted under strict observance of the principles of the Declaration of Helsinki. All patients had originally been referred for endoscopic evaluation because of one or more of the following symptoms: dysphagia, heartburn, noncardiac chest pain, and regurgitation. Forty-tree specimens were classified as intestinal metaplasia and two as low-grade dysplasia.

\section{Tissue specimens and Immunohistochemistry (IHC) Staining}

Endoscopic (mucosal) biopsy specimens from BE patients and matched tumor and adjacent normal specimens from EC patients, archived as formalin-fixed, paraffin-embedded material, were used for immunohistochemical analysis. Sections were routinely stained with hematoxylin and eosin and evaluated histopathologically. Tissue specimens were processed in a standard fashion, as described 
elsewhere [22]. ENO1 was identified using an anti-ENO1 mouse monoclonal antibody at a concentration of $0.025 \mu \mathrm{g} / \mathrm{ml}$ (Abcam, Cambridge, UK). Immunostaining was with a peroxidase-based visualization DAKO LSAB $^{\circledR}$ kit, following the manufacturer's recommendations. Diaminobenzidine tetrahydrochloride was used as chromogen. Sections incubated with PBS instead of the anti-ENO1 antibody served as controls.

\section{Evaluation of staining}

Histopathological examination was done by a senior pathologist (L.C.) who was unaware of the clinical findings. ENO1 expression into the cytoplasm and nucleus was empirically determined. IHC staining was graded using two semiquantitative measurements: staining intensity $(0-4)$ and percentage of cells stained ( $0=$ no staining, $1=$ below $25 \%, 2=25 \%-50 \%, 3=50 \%-75 \%$, and $4=75 \%-100 \%)$. A combined immunoreactive score (IRS) [23] was calculated as the product of staining intensity and percentage of stained cells.

\section{Determination of plasma ENOI levels by ELISA}

Peripheral blood samples were collected by aspiration in Vacutainer tubes containing 0.105 mol/liter sodium citrate, from EC patients $(n=32)$ before surgery, BE patients $(n=19)$, and healthy subjects $(n=25)$. Blood was then centrifuged and plasma were aliquoted and stored at $-80^{\circ} \mathrm{C}$ until use. ENO1 concentrations were determined using a specific ELISA kit (USCN Life Science Inc., Houston, TX 77082, USA). The lower detection limit of the assay was $39 \mathrm{pg} / \mathrm{ml}$.

\section{Statistical analysis}

Statistical analysis was performed using the GraphPad Prism 7 package (GraphPad Software, La Jolla, CA, USA). The Wilcoxon signed rank test and Mann-Whitney Rank Sum Test or Student's $t$ test were used to evaluate statistically significant differences between datasets. The relationships between variables were investigated by the Spearman or Pearson correlation tests. One Way Analysis of Variance or Kruskal-Wallis One Way Analysis of Variance on Rank followed by All Pairwise Multiple Comparison Procedures (Dunn's Method) were applied to determine whether significant differences existed among the study groups. In determining the correlation between ENO1 tissue expression or plasma levels and prognosis, the median IRS or ENO1 concentration in the peripheral blood was taken as cut-off. Survival data were analyzed using the
Kaplan-Meier method with a log-rank test for comparison. $P<0.05$ was considered to indicate a statistically significant difference.

\section{Results}

\section{ENO1 expression was up-regulated in EC in comparison with adjacent non-cancerous tissue samples}

In situ ENO1 protein expression status was analyzed by IHC in matched EC and adjacent normal tissue specimens $(n=40)$. In tumor cells, positive expression was detected in $80 \%$ of EC patients, whereas in paired normal counterparts only $10 \%$ were positive. When patients were categorized by histological tumor subtype, ENO1 was expressed in tumor cells in $76.9 \%$ of ESCC cases and in $81.5 \%$ in EAC cases, and in matched normal tissues in $7.8 \%$ and $11.1 \%$, respectively.

The semiquantitative assessment of staining (IRS) demonstrated markedly higher level of ENO1 in the clinical tumor samples of EC than in the adjacent normal tissues [IRS median (range): 6 (0-9) vs. 0 (0-4), $p<0.0001$ ] (Figure 1).

Figure 2A shows representative images of immunohistochemical staining in ESCC $(n=13)$, EAC $(n=27)$ and the relative adjacent non-tumor tissue specimens. There was no significant difference between ESCC and EAC in either the tumor area or the adjacent normal tissue, with predominant strong IHC staining in the cytoplasm of basal cells and/or squamous epithelium [IRS median (range): ESCC $=6$ $(0-9)$ vs. $\mathrm{EAC}=6$ (0-9), $p=0.5945$ and IRS median (range): ESCC normal adjacent tissue $=0(0-4)$ vs. EAC normal adjacent tissue $=0(0-4), p=0.8029]$ (Figure 2B).

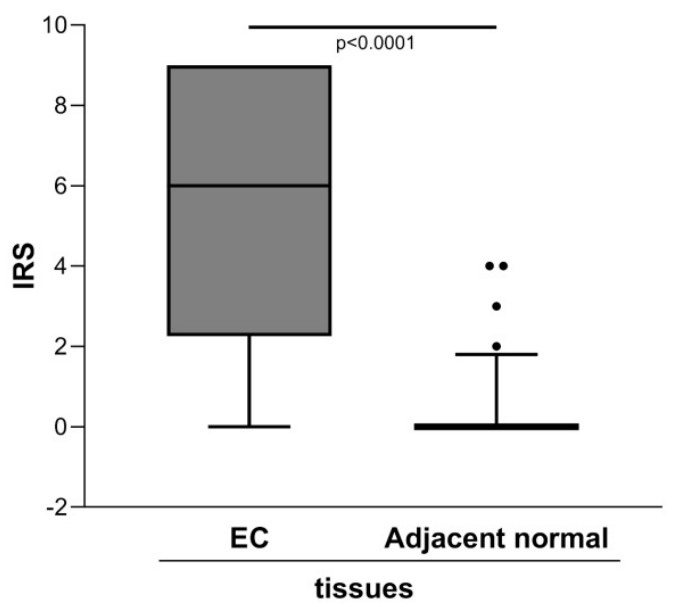

Figure 1. Quantitative immunohistochemical analysis of ENO1 protein expression in paired EC and normal adjacent tissues samples $(n=40)$. The immunoreactive scores (IRS) were obtained as described in the Materials and Methods section. Median, 10th, $25^{\text {th }}, 7^{\text {th }}$, and $90^{\text {th }}$ percentiles are presented as vertical boxes with error bars. Dots indicate outliers. $P$-values obtained by Wilcoxon signed rank test. 
A

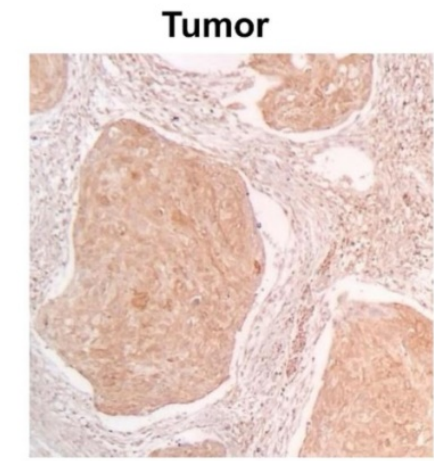

Tumor

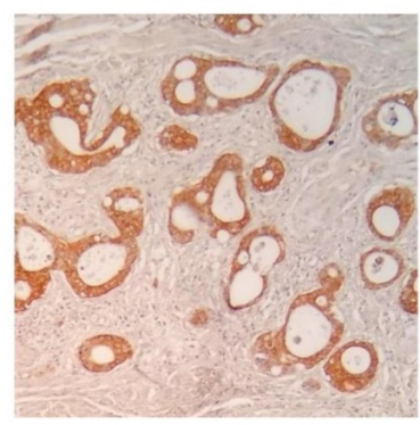

B

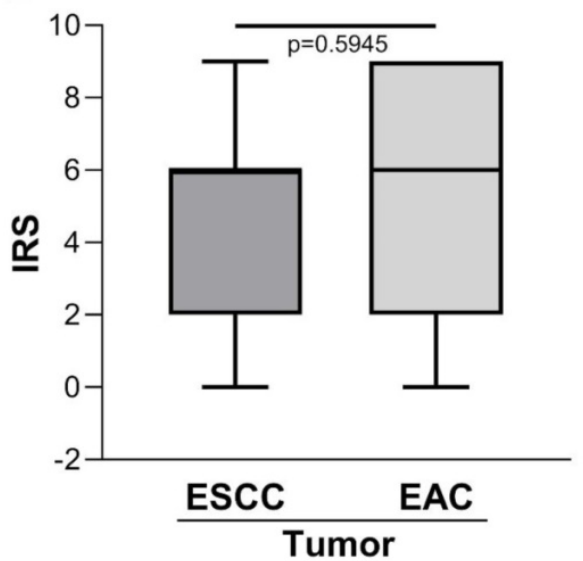

Adjacent normal tissue

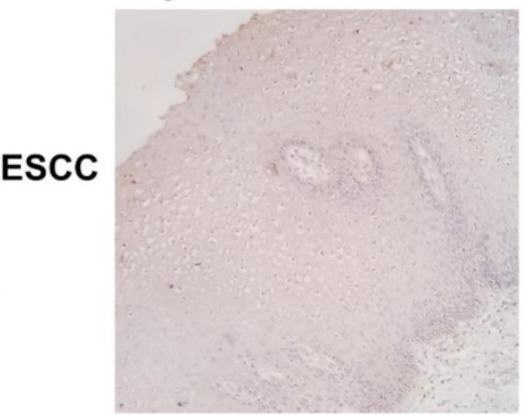

Adjacent normal tissue

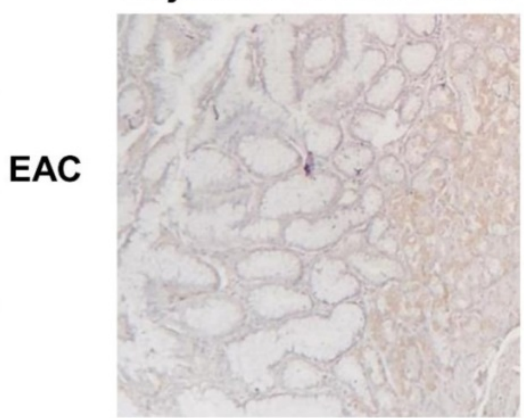

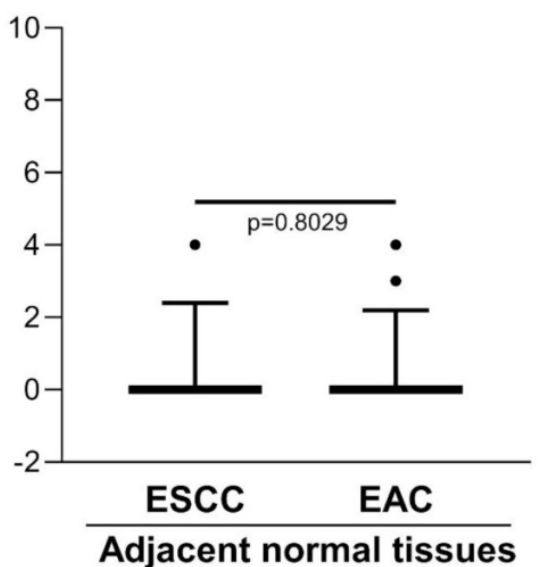

Figure 2. (A) Representative immunohistochemical analysis of ENOI protein expression in ESCC and EAC tissues specimens and in paired normal adjacent esophagus mucosa sections (original magnification x 100). No staining in negative controls (data not shown). (B) Quantitative analysis of immunostaining for ENO1 protein in ESCC, EAC and paired normal adjacent tissues samples $(n=40)$. The immunoreactive scores (IRS) were obtained as described in the Materials and Methods section. Median, $10^{\text {th }}$, $25^{\text {th }}$, $75^{\text {th }}$, and $90^{\text {th }}$ percentiles are presented as vertical boxes with error bars. Dots indicate outliers. P-values obtained by Mann-Whitney Rank Sum Test.

\section{ENOI expression in BE versus EAC tissue samples}

Considering the known risk of $\mathrm{BE}$ progression toward EAC, we also evaluated ENO1 protein expression in BE $(n=45)$ and in unrelated EAC tissue samples by IHC, and compared the two. In BE cases, ENO1 expression was fairly homogeneous in squamous epithelium and in areas of intestinal metaplasia, with intense cytoplasmic staining in the specialized gastric fundus cells. Representative examples of staining in BE $(n=45)$ and EAC $(n=27)$ tissues are shown in Figure 3A. ENO1 IRS in BE tissues was lower than in unrelated EAC tissues [IRS median (range): $\mathrm{BE}=2(0-9)$ vs. $\mathrm{EAC}=6(0-9), p=0.0025]$
(Figure 3B).

\section{Correlation between tissue ENOI expression and clinicopathological parameters in EC}

Local ENO1 protein expression in EC was not associated with sex, age or tumor location $(r=0.209$, $p=0.1942, \mathrm{r}=0.259, p=0.1060$, and $\mathrm{r}=-0.197, p=0.2210$, respectively, Spearman correlation test). When EC cases were classified by degree of tumor differentiation, no statistically significant difference in ENO1 expression was found between grade $2(n=14)$ (IRS mean $\pm S E$ : $5.93 \pm 0.77)$, grade $2 / 3 \quad(n=10)$ (IRS mean \pm SE: $3.84 \pm 1.21)$, and grade 3 tumors $(n=16)$ (IRS mean \pm SE: $3.46 \pm 0.87$ ) (One Way Analysis of Variance $p=0.4671)$ (data not shown). 
A

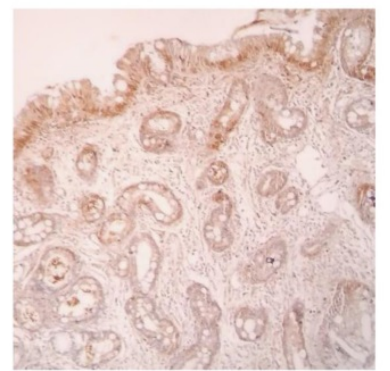

BE

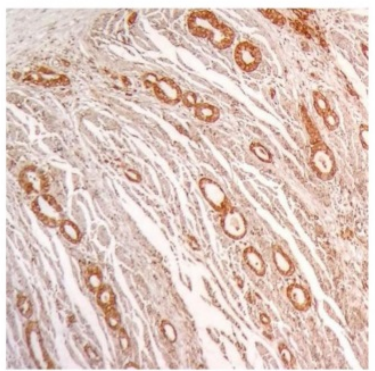

EAC
B

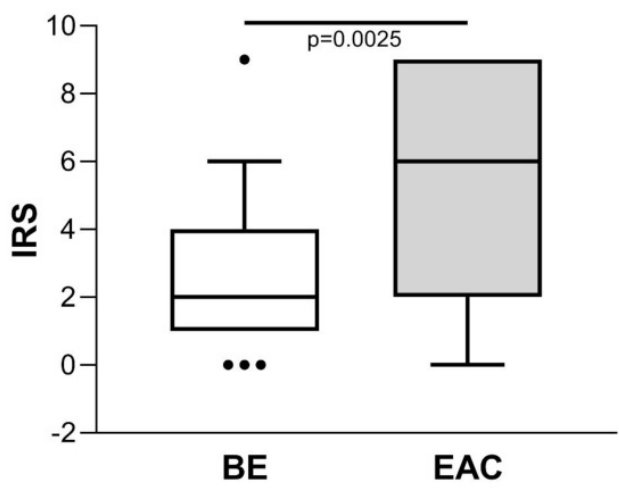

Figure 3. (A) Representative immunohistochemical analysis of ENOI protein expression in $\mathrm{BE}$ and unrelated EAC tissues specimens (original magnification $\times 100$ ). No staining in negative controls (data not shown). (B) Quantitative analysis of immunostaining for ENOI protein in BE and unrelated EAC tissue samples. The immunoreactive scores (IRS) were obtained as described in the Materials and Methods section. Median, $10^{\text {th }}, 25^{\text {th }}, 7^{\text {th }}$, and $90^{\text {th }}$ percentiles are presented as vertical boxes with error bars. Dots indicate outliers. P-values obtained by Mann-Whitney Rank Sum Test.

Conversely, for the IRS of tumor cases stratified by disease stage, there was a statistical significant increasing trend towards the later stages [IRS median (range): stage I + II A/B=3 (0-9), stage III A/B=6 (0-9), stage IV=9 (6-9); $p=0.0005$ Kruskal-Wallis One Way Analysis of Variance on Rank; stage I + IIA/B vs. stage III A/B, $p=0.0219$; stage I + II A/B vs. stage IV, $p=0.010$; stage III A/B vs. stage IV, $p=0.3234$, Dunn's multiple comparisons test] (Figure 4A). No significant association was found between ENO1 expression quantified by IRS and EC stage in paired adjacent normal tissues $(p=0.3813$, Kruskal-Wallis One Way Analysis of Variance on Rank) (data not shown). When EC was classified according to the two major histologic types, differences in IRS values of the ESCC disease stage II A/B vs. III + IV, and EAC disease stage I + II A/B vs. III + IV were statistically significant [IRS mean \pm SE: $2.333 \pm 1.085$ vs. $6.571 \pm 0.685$, $p=0.0059$, and IRS median (range): 3 (0-9) vs. 9 (0-9), $p=0.0214$, respectively] (Figure 4B and C).
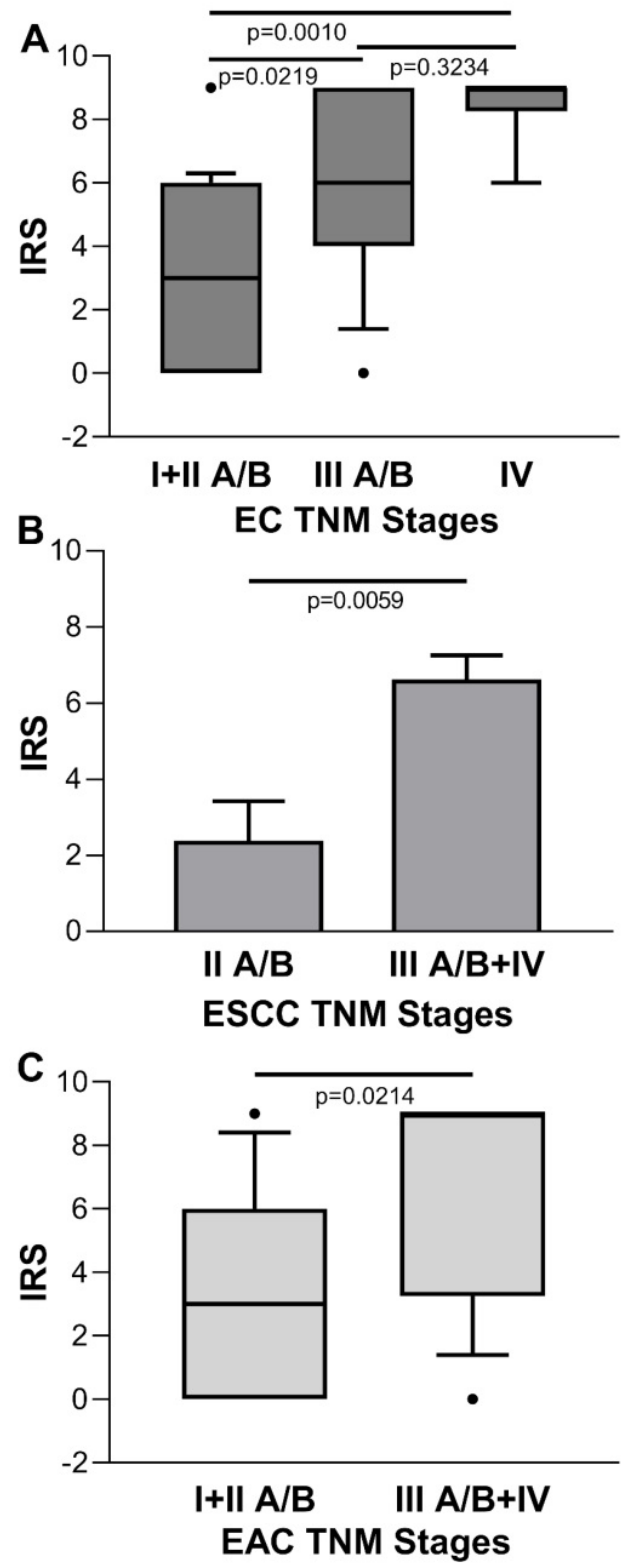

Figure 4. (A) Quantitative analysis of immunostaining for ENO1 protein in tissue samples from EC categorized by disease stage. Median, 10th, 25th, 75th, and $90^{\text {th }}$ percentiles are presented as vertical boxes with error bars. Dots indicate outliers. $P$-values obtained by Kruskal-Wallis One Way Analysis of Variance on Ranks followed by All Pairwise Multiple Comparison Procedures (Dunn's Method). (B) Quantitative analysis of immunostaining for ENOI protein in tissue samples from ESCC categorized by disease stage. Results are expressed as mean $\pm S E$. $P$-values obtained by Student's $t$ test. (C) Quantitative analysis of immunostaining for ENOI protein in tissue samples from EAC categorized by disease stage. Median, $10^{\text {th }}, 25^{\text {th }}, 75^{\text {th }}$, and $90^{\text {th }}$ percentiles are presented as vertical boxes with error bars. Dots indicate outliers. $P$-values obtained by Mann-Whitney Rank Sum Test. The immunoreactive scores (IRS) were obtained as described in the Materials and Methods section.

\section{ENO1 was down-regulated in plasma of EAC patients}

The mean \pm SE ENO1 level in plasma of the EC group $(\mathrm{n}=32)$ was $2183 \pm 171 \mathrm{pg} / \mathrm{ml}$, which was significantly below the concentration found in healthy donors $(\mathrm{n}=25) \quad(3693 \pm 246 \mathrm{pg} / \mathrm{ml}, p<0.0001)$ (Figure $5 \mathrm{~A})$. When EC cases were classified by histological type, in ESCC patients $(n=8)$ circulating ENO1 
concentrations did not differ from those in normal subjects (mean \pm SE pg/ml: $2849 \pm 309$ vs $3693 \pm 246$, $p=0.083$ ), but were significantly higher than in EAC patients $\quad(\mathrm{n}=24) \quad($ mean $\pm \mathrm{SE} \quad \mathrm{pg} / \mathrm{ml}: \quad 2849 \pm 309$ vs $1961 \pm 185, p=0.022)$. By contrast, in the EAC group plasma ENO1 levels were significantly lower than those in healthy donors (mean \pm SE pg/ml: 1961 \pm 185 vs $3693 \pm 246, p<0.0001)$.
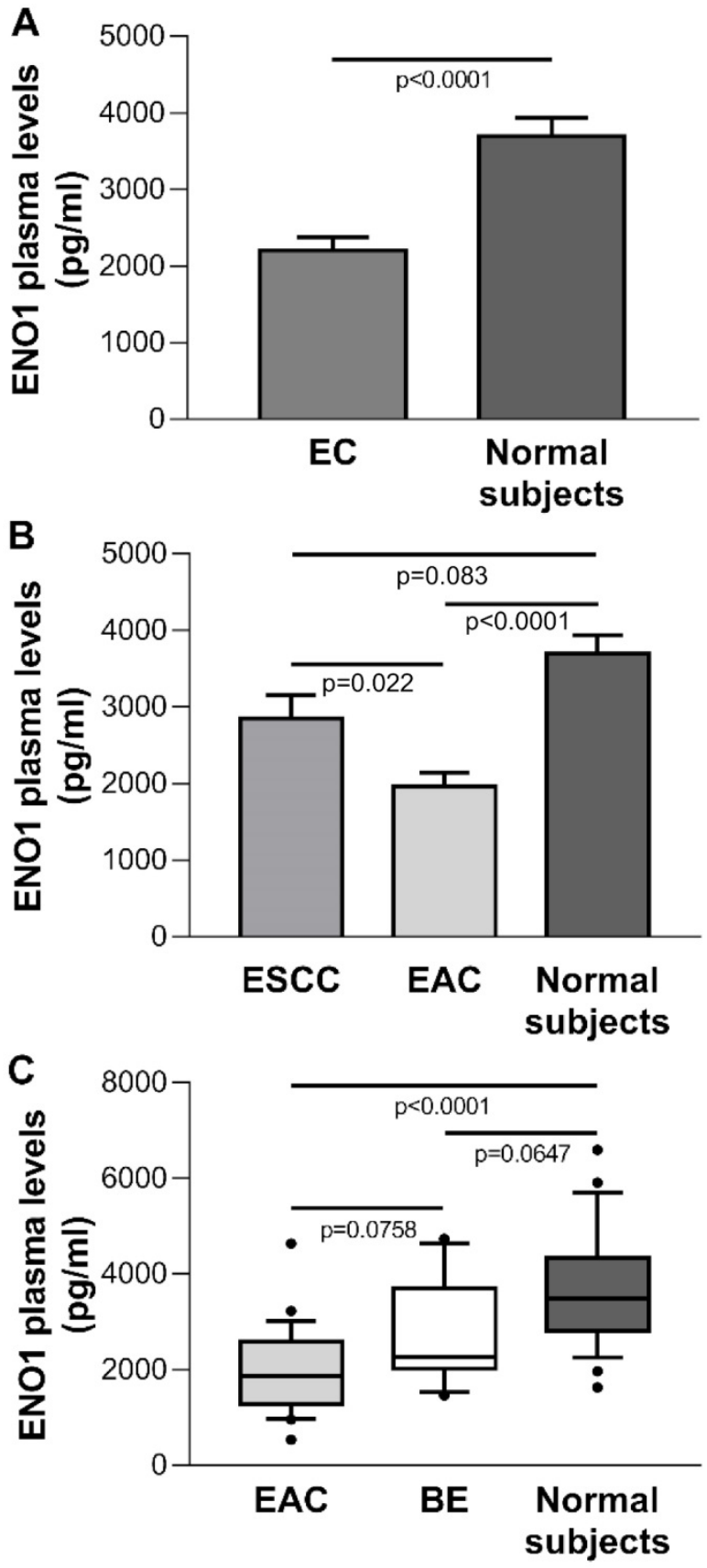

Figure 5. (A) Plasma ENOI concentration by ELISA in EC patients and normal subjects. P-values obtained by Student's $t$ test. (B) Plasma ENOI concentration by ELISA in EC patients categorized by histological type of tumor (ESCC and EAC) and normal subjects. Results are expressed as mean \pm SE. ENOI concentration $(\mathrm{pg} / \mathrm{ml})$. $P$-values obtained by Unpaired Studenti's t-test. (C) Plasma ENOI concentration by ELISA in normal subjects, BE and EAC patients. Median, $10^{\text {th }}, 25^{\text {th }}, 75^{\text {th }}$, and $90^{\text {th }}$ percentiles are presented as vertical boxes with error bars. Dots indicate outliers. $P$-values obtained by Kruskal-Wallis One Way Analysis of Variance on Ranks followed by All Pairwise Multiple Comparison Procedures (Dunn's Method).

\section{ENOI plasma levels in BE and EAC patients}

To determine whether circulating ENO1 may be an important risk factor for progression from $\mathrm{BE}$ to $E A C$, the plasma concentrations of this enzyme in normal subjects, BE cases $(\mathrm{n}=19)$, and EAC patients were compared. A trend toward increased ENO1 levels was found in the sequence EAC patients, BE patients, healthy subjects [median (range) pg/ml: 1867 (538-4636), 2273 (1461-4737), 3494 (1629-6596), respectively, $p<0.0001$, Kruskal-Wallis One Way Analysis of Variance on Ranks; EAC vs. BE, $p=0.0758$; EAC vs. normal donors, $p<0.0001$; BE vs. normal donors, $p=0.0647$ (Dunn's multiple comparisons test)] (Figure 5C).

\section{Correlation between circulating ENOI level and clinicopathological parameters in EC}

ENO1 plasma levels in EC was not associated with age, sex, or tumor location $(\mathrm{r}=-0.1093, \mathrm{p}=0.5515$, Pearson correlation test; $\mathrm{r}=-0.1393, \mathrm{p}=0.4472$, and $\mathrm{r}=0.2158, \mathrm{p}=0.235$, Spearman correlation test, respectively). When EC cases were classified by degree of tumor differentiation, no statisticallysignificant difference in circulating ENO1 levels was found between grade $2(n=11)$, grade $2 / 3(n=7)$, and grade 3 tumors $(\mathrm{n}=14)$ (mean $\pm \mathrm{SE} \mathrm{pg} / \mathrm{ml}, 2143 \pm 178$, $2161 \pm 384,2225 \pm 326$, respectively; $p=0$. 9773, One Way Analysis of Variance), even when EC cases were categorized by histological type (data not shown).

Further, when plasma levels of EC cases were stratified by disease stage, there was no statistically significant difference [mean $\pm \mathrm{SE} \mathrm{pg} / \mathrm{ml}: 2346 \pm 251$, stage I + II/B $(n=15) ; 2266 \pm 306$, stage III A/B $(n=11)$; $1624 \pm 324$, stage IV $(n=6) ; p=0.2940$, One Way Analysis of Variance], even when EC cases were categorized by histological type (data not shown).

\section{Overall survival analysis}

To define the clinical significance of local and circulating ENO1 protein levels in EC, the correlation between ENO1 IRS and plasma concentrations and overall survival was analyzed. The Kaplan-Meier curves for overall survival of all EC patients showed that neither parameter was significantly associated with survival (ENO1 IRS and plasma concentration: $p=0.7258$ and $p=0.8501$, respectively), even when EC cases were subdivided by histological type (ESCC ENO1 IRS and plasma concentration: $p=0.1125$ and $p=0.5835$, respectively; EAC ENO1 IRS and plasma concentration: $p=0.3739$ and $p=0.5328$, respectively).

\section{Discussion}

ENO1, as a key glycolytic enzyme, may play pivotal role in aerobic glycolysis (the so-called Warburg effect) contributing to tumor progression of 
numerous cancers.

The present study targeted the hitherto unexamined expression of ENO1 protein, using an immunohistochemical method, in tumor and matched adjacent normal tissue sections from EC patients, as well as ENO1 circulating levels; their clinical relevance was also examined. It showed that: i) ENO1 production is abnormally elevated in cancer cells in both ESCC and EAC, increasing during tumor stage progression and in non-dysplastic $\mathrm{BE}$, the premalignant condition that predisposes to the development of EAC, ii), unexpectedly, EAC patients exhibited significantly lower ENO1 plasma levels compared to normal subjects, and iii) both in situ and systemic ENO1 levels showed no significant correlation with overall survival of the patients. These results suggest that upregulation of ENO1 may accelerate the cells' glucose metabolism in esophagus cells as early event in the Barrett's adenocarcinoma transition and in association with tumor progression.

In agreement with numerous studies on different human cancer types [13-19, 24-30], the study found that ENO1 protein was up-regulated in EC tissues in comparison with adjacent non-tumorous tissues.

In addition to glycolytic activity, ENO1 appears to have various cellular functions and subcellular localizations, performing important role in other pathophysiological processes in cancer [31]. When located in the nucleus, the enzyme may inhibit transcription of the proto-oncogene c-myc, acting as a transcriptional repressor [32]. Moreover, ENO1 expressed on the cell surface can function as a plasminogen receptor, and contributes to cell invasion and metastasis [33]. In the cytoplasm, ENO1 provides a rapidly available supply of ATP to cells and, interacting with the cytoskeletal system, supports the rapid growth, proliferation, and movement of cancer cells [20]. ENO1 also strengthens the infiltration ability of monocytes and macrophages, and it can participate in tumor formation by controlling the expression of the c-myc oncoprotein through the Notch signaling pathway [34].

IHC analysis failed to detect enhanced ENO1 membrane or nuclear localization in EC tissue specimens, indicating that the enzyme activity is likely involved in regulating glycolysis rather than in controlling transcription and/or extracellular matrix remodeling.

EC includes two main subtypes: ESCC, which develops from squamous epithelium undergoing inflammatory, hyperplastic, and dysplastic changes, and EAC, which arises through metaplastic intestinal-type changes replacing the squamous epithelium. The study found that ENO1 in situ protein levels did not differ significantly between ESCC and
EAC patients, suggesting that ENO1 expression is not associated with the pathological tumor type. Moreover, statistical analysis revealed that high ENO1 immunohistochemical expression levels in both ESCC and EAC were significantly associated with disease stage, but not with other clinical features such as age, gender, tumor location, or degree of tumor differentiation. A positive correlation has been reported between elevated in situ ENO1 protein expression and cancer progression in pancreatic and hepatocellular carcinoma patients [25, 28]. By contrast, in lung, colon, and nasopharyngeal cancer tissues, ENO1 expression appears inversely correlated with disease stage $[29,35]$. These results suggest that ENO1 may play different roles in tumor growth, depending on the type of cancer, although specific action mechanisms remain unclear.

$\mathrm{BE}$ is considered a complication of chronic gastroesophageal reflux, and represents a major risk factor for development of EAC. Currently, alongside dysplasia, few molecular markers may be used to delineate a population of $\mathrm{BE}$ patients at high risk for cancer $[36,37]$. In the present study, ENO1 protein was found to be in situ overexpressed already in non-dysplastic BE, as well as in EAC compared to normal surrounding mucosa, with a progressive increase along the sequence normal, premalignant and neoplastic epithelium. ENO1 might thus be a useful biomarker of development of BE in EAC, although further studies will be needed to fully document the diagnostic and prognostic value of ENO1 in this process.

Although ENO1 has been extensively evaluated using proteomics and IHC in tumor cell lines and tissues, few studies have examined circulating levels of the enzyme in cancer patients. ENO1 may be delivered into bloodstream by tumor cell necrosis and turnover or unconventional secretory pathways. Abnormally higher circulating ENO1 levels were reported in non-small-cell lung cancer (NSCLC) [38] and pancreatic cancer patients [25]. Unexpectedly, we found that EAC, but not ESSC patients had in plasma significantly lower ENO1 level compared with healthy individuals. Interestingly, circulating ENO1 levels decreased progressively in normal, precancerous condition of the esophagus and EAC sequence, exactly in the opposite way to the tissue expression of the protein. Therefore, also in the periphery, BE mucosal abnormality, a clearly recognized risk factor for EAC development, is associated with altered plasmatic ENO1 levels that may predate the clinical diagnosis of malignancy.

It is tempting to speculate that the increase in glucose uptake and elevated proliferation rates associated with the in situ tumor growth may provoke 
a rapid consumption of ENO1, the master regulator of tumor metabolism, and in turn an aberrant release of the enzyme. Otherwise, circulating ENO1 can be degraded by some activated proteases secreted from tumor or released from tumor cell death [39]. Moreover, it is well documented that ENO1 might act as an autoantigen in several cancer [40,41], reflecting the greater immunologic reactivity and enhanced immune surveillance for cancer cells.

ESSC and EAC are clearly distinct cancers with differences in risk factors, histopathology and molecular profile. Tumor microenvironment could potentially drive this divergence. Since the tissue expression of the protein ENO1 did not differ significantly in the two EC subtypes, a potential mechanism accounting for decreased levels of circulating ENO1 in EAC patients could be a more prompt clearance of the immunocomplexes formed by ENO1/anti-ENO1 antibodies, released into the circulation during tumor growth. However, taking into account the discrepancies between our results and those of other studies, the clinical significance of circulating ENO1 in EC requires further exploration, including the quantification of the production of ENO1 autoantibodies.

Previous investigations report an association between in situ ENO1 overexpression and a worse clinical outcome in a variety of tumors, such as pancreatic adenocarcinoma [25], hepatocellular carcinoma [28, 42], glioma [14], breast cancer [15], and head and neck cancer [17]. Conversely, most NSCLC patients, who show down-regulated ENO1 expression, exhibit a poor prognosis [16]. It emerged from the present study that, in EC, local ENO1 protein upregulation was related only to tumor progression and not to overall survival. The different findings reported by other studies and the present work may be related to the types of cells investigated, and might also be closely related to ENO1 expression level.

As reported above, besides its innate glycolytic function, ENO1 may play other roles in tumorigenesis depending on its cellular localization. This study found that ENO1 in EC cells was mainly localized in the cytoplasm, consistent with its supporting role in the high metabolic rate of tumor cells. As occurs in breast cancer and NSCLC [15, 16, 43], ENO1 surface and nuclear forms may be involved in cancer invasion and metastasis, as well as in transcriptional repression, apparently inhibiting cell growth and accelerating apoptosis and necrosis [44]. Moreover, in gastric cancer, ENO1 protein levels are associated with chemoresistance [45]. Thus, the various cellular localizations, as well as the different pathways regulating ENO1 functions in the different types of tumor cells, may account for the inconsistence of the experimental results reported thus far.

In conclusion, alongside this explorative study, future in-depth investigations are needed to further elucidate the specific clinical value of ENO1 in EC, in consideration of its possible utility in delineating a population of patients with BE at high risk of cancer, and representing a new therapeutic target to counteract the growth and progression of this aggressive tumor.

\section{Abbreviations}

BE: Barrett's esophagus; EAC: esophageal adenocarcinoma; EC: esophageal cancer; ENO: Enolase; ESCC: esophageal squamous cell carcinoma; IHC: immunohistochemistry; IRS: immunoreactive score; NSCLC: non-small-cell lung cancer; TNM: tumor node metastasis.

\section{Acknowledgements}

This work was supported by grants from the Piedmontese Regional Government (Regione Piemonte) and the World Wide Style (WWS) Project, University of Turin and CRT Foundation, Italy, to MC. AHT was the recipient of an award from the University of Turin and CRT Foundation-WWS Project, Italy.

We thank Madam Adriana Prati for providing the group technical assistance.

\section{Competing Interests}

The authors have declared that no competing interest exists.

\section{References}

1. Siegel RL, Miller KD, Jemal A. Cancer statistics, 2018. CA Cancer J Clin. 2018; 68: 7-30.

2. Lagergren J, Smyth E, Cunningham D, Lagergren P. Oesophageal cancer. Lancet. 2017; 390: 2383-96.

3. Shapiro J, van Lanschot JJB, Hulshof MCCM, van Hagen P, van Berge Henegouwen MI, Wijnhoven BPL, et al. Neoadjuvant chemoradiotherapy plus surgery versus surgery alone for oesophageal or junctional cancer (CROSS): long-term results of a randomised controlled trial. Lancet Oncol. 2015; 16: 1090-8.

4. Klein CA, Stoecklein NH. Lessons from an aggressive cancer: evolutionary dynamics in esophageal carcinoma. Cancer Res. 2009; 69: 5285-8.

5. Wang K, Johnson A, Ali SM, Klempner SJ, Bekaii-Saab T, Vacirca JL, et al. Comprehensive Genomic Profiling of Advanced Esophageal Squamous Cell Carcinomas and Esophageal Adenocarcinomas Reveals Similarities and Differences. Oncologist. 2015; 20: 1132-9.

6. Jain S, Dhingra S. Pathology of esophageal cancer and Barrett's esophagus. Ann Cardiothorac Surg. 2017; 6: 99-109.

7. Siewert JR, Ott K. Are squamous and adenocarcinomas of the esophagus the same disease? Semin Radiat Oncol. 2007; 17: 38-44.

8. Kang HJ, Jung SK, Kim SJ, Chung SJ. Structure of human alpha-enolase (hENO1), a multifunctional glycolytic enzyme. Acta Crystallogr D Biol Crystallogr. 2008; 64: 651-7.

9. Díaz-Ramos A, Roig-Borrellas A, García-Melero A, López-Alemany R. a-Enolase, a multifunctional protein: its role on pathophysiological situations. J Biomed Biotechnol. 2012; 2012: 156795.

10. Chang GC, Liu KJ, Hsieh CL, Hu TS, Charoenfuprasert S, Liu HK, et al. Identification of alpha-enolase as an autoantigen in lung cancer: its overexpression is associated with clinical outcomes. Clin Cancer Res. 2006; 12: 5746-54. 
11. López-Pedrera C, Villalba JM, Siendones E, Barbarroja N, Gómez-Díaz C, Rodríguez-Ariza A, et al. Proteomic analysis of acute myeloid leukemia: Identification of potential early biomarkers and therapeutic targets. Proteomics. 2006; 6 Suppl 1: S293-9.

12. Katayama M, Nakano H, Ishiuchi A, Wu W, Oshima R, Sakurai J, et al. Protein pattern difference in the colon cancer cell lines examined by two-dimensional differential in-gel electrophoresis and mass spectrometry. Surg Today. 2006; 36: 1085-93.

13. Bai Z, Ye Y, Liang B, Xu F, Zhang H, Zhang Y, et al. Proteomics-based identification of a group of apoptosis-related proteins and biomarkers in gastric cancer. Int J Oncol. 2011; 38: 375-83.

14. Song $\mathrm{Y}$, Luo Q, Long $\mathrm{H}, \mathrm{Hu} \mathrm{Z}$, Que T, Zhang X, et al. Alpha-enolase as a potential cancer prognostic marker promotes cell growth, migration, and invasion in glioma. Mol Cancer. 2014; 13: 65.

15. Tu SH, Chang CC, Chen CS, Tam KW, Wang YJ, Lee CH, et al. Increased expression of enolase alpha in human breast cancer confers tamoxifen resistance in human breast cancer cells. Breast Cancer Res Treat. 2010; 121: $539-53$

16. Fu QF, Liu Y, Fan Y, Hua SN, Qu HY, Dong SW, et al. Alpha-enolase promotes cell glycolysis, growth, migration, and invasion in non-small cell lung cancer through FAK-mediated PI3K/AKT pathway. J Hematol Oncol. 2015; 8: 22

17. Tsai ST, Chien IH, Shen WH, Kuo YZ, Jin YT, Wong TY, et al. ENO1, a potential prognostic head and neck cancer marker, promotes transformation partly via chemokine CCL20 induction. Eur J Cancer. 2010; 46: 1712-23.

18. Zhao M, Fang W, Wang Y, Guo S, Shu L, Wang L, et al. Enolase-1 is a therapeutic target in endometrial carcinoma. Oncotarget. 2015; 6: 15610-27.

19. Principe M, Ceruti P, Shih NY, Chattaragada MS, Rolla S, Conti L, et al. Targeting of surface alpha-enolase inhibits the invasiveness of pancreatic cancer cells. Oncotarget. 2015; 6: 11098-113.

20. Vander Heiden MG, Cantley LC, Thompson CB. Understanding the Warburg effect: the metabolic requirements of cell proliferation. Science. 2009; 324: 1029-33.

21. Rice TW, Patil DT, Blackstone EH. 8th edition AJCC/UICC staging of cancers of the esophagus and esophagogastric junction: application to clinical practice. Ann Cardiothorac Surg. 2017; 6: 119-30.

22. Vizio B, Boita M, Cristiano C, Mazibrada J, Bosco O, Novarino A, et al. Thymic stromal lymphopoietin in human pancreatic ductal adenocarcinoma: expression and prognostic significance. Oncotarget. 2018; 9: 32795-809.

23. Remmele W, Stegner HE. [Recommendation for uniform definition of an immunoreactive score (IRS) for immunohistochemical estrogen receptor detection (ER-ICA) in breast cancer tissue]. Pathologe. 1987; 8: 138-40.

24. Niccolai E, Cappello P, Taddei A, Ricci F, D'Elios MM, Benagiano M, et al. Peripheral ENO1-specific $\mathrm{T}$ cells mirror the intratumoral immune response and their presence is a potential prognostic factor for pancreatic adenocarcinoma. Int J Oncol. 2016; 49: 393-401.

25. Yin H, Wang L, Liu HL. ENO1 Overexpression in Pancreatic Cancer Patients and Its Clinical and Diagnostic Significance. Gastroenterol Res Pract. 2018; 2018: 3842198

26. Liu YQ, Huang ZG, Li GN, Du JL, Ou YP, Zhang XN, et al. Effects of a-enolase (ENO1) over-expression on malignant biological behaviors of AGS cells. Int J Clin Exp Med. 2015; 8: 231-9.

27. Zhan $\mathrm{P}$, Zhao $\mathrm{S}$, Yan $\mathrm{H}$, Yin $\mathrm{C}$, Xiao $\mathrm{Y}$, Wang $\mathrm{Y}$, et al. a-enolase promotes tumorigenesis and metastasis via regulating AMPK/mTOR pathway in colorectal cancer. Mol Carcinog. 2017; 56: 1427-37.

28. Zhu W, $\mathrm{Li} \mathrm{H}, \mathrm{Yu}$ Y, Chen J, Chen X, Ren F, et al. Enolase-1 serves as a biomarker of diagnosis and prognosis in hepatocellular carcinoma patients. Cancer Manag Res. 2018; 10: 5735-45.

29. Zhang L, Wang H, Dong X. Diagnostic value of a-enolase expression and serum a-enolase autoantibody levels in lung cancer. J Bras Pneumol. 2018; 44: $18-23$

30. Zhu X, Miao X, Wu Y, Li C, Guo Y, Liu Y, et al. ENO1 promotes tumor proliferation and cell adhesion mediated drug resistance (CAM-DR) in Non-Hodgkin's Lymphomas. Exp Cell Res. 2015; 335: 216-23.

31. Ji H, Wang J, Guo J, Li Y, Lian S, Guo W, et al. Progress in the biological function of alpha-enolase. Anim Nutr. 2016; 2: 12-7.

32. Ray R, Miller DM. Cloning and characterization of a human c-myc promoter-binding protein. Mol Cell Biol. 1991; 11: 2154-61.

33. Pancholi V. Multifunctional alpha-enolase: its role in diseases. Cell Mol Life Sci. 2001; 58: 902-20.

34. Hsu KW, Hsieh RH, Lee $\mathrm{YH}$, Chao $\mathrm{CH}, \mathrm{Wu} \mathrm{KJ}$, Tseng MJ, et al. The activated Notch1 receptor cooperates with alpha-enolase and MBP-1 in modulating c-myc activity. Mol Cell Biol. 2008; 28: 4829-42.

35. Cheng C, Long X, Li X, Xie M, Guo M. [The expressions of alpha-enolase in the nasopharyngeal cancer tissue]. Lin Chung Er Bi Yan Hou Tou Jing Wai Ke Za Zhi. 2011; 25: 554-6.

36. Naini BV, Souza RF, Odze RD. Barrett's Esophagus: A Comprehensive and Contemporary Review for Pathologists. Am J Surg Pathol. 2016; 40: e45-66.

37. Bennett $M$, Mashimo $H$. Molecular markers and imaging tools to identify malignant potential in Barrett's esophagus. World J Gastrointest Pathophysiol. 2014; 5: 438-49.

38. ZHANG Y, LI M, LIU Y, HAN N, ZHANG K, XIAO T, et al. [ENO1 protein levels in the tumor tissues and circulating plasma samples of non-small cell lung cancer patients]. Zhongguo Fei Ai Za Zhi. 2010; 13: 1089-93.
39. Villanueva J, Shaffer DR, Philip I, Chaparro CA, Erdjument-Bromage $\mathrm{H}_{\text {, }}$ Olshen $\mathrm{AB}$, et al. Differential exoprotease activities confer tumor-specific serum peptidome patterns. J Clin Invest. 2006; 116: 271-84.

40. Sato N, Nabeta Y, Kondo H, Sahara H, Hirohashi Y, Kashiwagi K, et al. Human CD8 and CD4 T cell epitopes of epithelial cancer antigens. Cancer Chemother Pharmacol. 2000; 46 Suppl: S86-90.

41. Fernández Madrid F. Autoantibodies in breast cancer sera: candidate biomarkers and reporters of tumorigenesis. Cancer Lett. 2005; 230: 187-98.

42. Hamaguchi T, lizuka N, Tsunedomi R, Hamamoto Y, Miyamoto T, Iida M, et al. Glycolysis module activated by hypoxia-inducible factor 1alpha is related to the aggressive phenotype of hepatocellular carcinoma. Int J Oncol. 2008; 33: 725-31.

43. Ghosh AK, Steele R, Ryerse J, Ray RB. Tumor-suppressive effects of MBP-1 in non-small cell lung cancer cells. Cancer Res. 2006; 66: 11907-12.

44. Feo S, Arcuri D, Piddini E, Passantino R, Giallongo A. ENO1 gene product binds to the c-myc promoter and acts as a transcriptional repressor: relationship with Myc promoter-binding protein 1 (MBP-1). FEBS Lett. 2000; 473: 47-52

45. Qian X, Xu W, Xu J, Shi Q, Li J, Weng Y, et al. Enolase 1 stimulates glycolysis to promote chemoresistance in gastric cancer. Oncotarget. 2017; 8: 47691-708. 